\title{
Optimization of Aqueous Nano Ceramic Ink and Printing Characterization for Digital Ink-Jet Printing
}

\author{
Jong-Woo Kwon****, Hee-Seok Sim*, Jong-Heun Lee**, Kwang-Taek Hwang*, \\ Kyu-Sung Han*, Jin-Ho Kim*, and Ung-Soo Kim*** \\ ${ }^{*}$ Ceramicware center, Korea Institute of Ceramic Engineering and Technology, Icheon 17303, Korea \\ **Department of Material Science and Engineering, Korea University, Seoul 02841, Korea
}

(Received June 1, 2017; Revised August 29, 2017; Accepted September 7, 2017)

\begin{abstract}
The advantage of ceramic ink-jet printing technology is the accurate and fast printing process of digital images for various products. For digital ink-jet printing applications, ceramic ink requires proper viscosity and surface tension, along with dispersion stability of the inorganic pigments. The purpose of this study is the formulation of an environment-friendly ceramic ink with a water-based system; using nano-sized $\mathrm{CoAl}_{2} \mathrm{O}_{4}$ pigment as a raw material, ink should have dispersion stability to prevent nozzle clogging during ink-jet printing process. In addition, the surface tension of the ceramic ink was optimized with the polysiloxane surfactant according to the surface tension requirement $(20-45 \mathrm{mN} / \mathrm{m})$ for ceramic ink-jet printing; by adjusting the viscosity with poly ethylene oxide, jetting behavior of the ceramic ink was investigated according to changes in the physical features through drop watcher measurement.
\end{abstract}

Key words : Suspensions, Traditional ceramics, Ink-jet printing, Raw material, Aqueous ceramic ink

\section{Introduction}

D igital ink-jet printing systems using nano-ceramic ink that stably develops color at high temperature have recently accomplished considerable technological advancements. ${ }^{1-2)}$ The ceramic inks used in the digital ink-jet printing technology generally require four primary colors, cyan, magenta, yellow, and black (CMYK), to realize full-color images. ${ }^{3)}$ In addition, nano-ceramic inks for inkjet printing, in order to be well jetted from a piezoelectric printer head, should have dispersion stability as well as an appropriate range of viscosity (4 to $40 \mathrm{mPa} \cdot \mathrm{s}$ ) and surface tension (20 to $45 \mathrm{mN} / \mathrm{m}) .^{3-8)}$

Ceramic inks currently used for digital printing are prepared using organic solvents that are relatively good at securing the dispersion stability of hydrophobic ceramic pigments. However, due to regulations on the volatile organic compounds (VOCs) contained in organic solvents and the life-shortening of inkjet heads by organic solvents, there is a growing interest in environment-friendly inkjet printing materials employing water-based ceramic inks. ${ }^{9-10)}$

In the present study, a nano-sized $\mathrm{CoAl}_{2} \mathrm{O}_{4}$ ceramic pigment was used to prepare a water-based environmentfriendly ceramic ink that has dispersion stability and shows good jetting characteristics when applied to an inkjet printing process. The drop formation behavior of the ceramic ink

\footnotetext{
Corresponding author: Ung-Soo Kim

E-mail : ukim@kicet.re.kr

Tel : +82-31-645-1422 Fax : +82-31-645-1422
}

was analyzed depending on the ceramic ink properties required by inkjet printing. In addition, the printing characteristics of the prepared ceramic ink were investigated on ceramic tiles.

\section{Experimental Procedure}

The properties of a blue ceramic pigment $\left(\mathrm{CoAl}_{2} \mathrm{O}_{4}\right.$, HANIL) applied to the ceramic inkjet printing process were evaluated by analyzing the crystal structure with an X-ray diffractometer (XRD, Rigaku, D/MAX2500VL/PC) and the particle shape and size through field emission scanning electron microscopy (FE-SEM, Jeol, JSM-6390). In addition, the BET (Brunauer-Emmett-Teller) surface area was analyzed using a BET surface measurement instrument (BET, TRISTA II 3200, Micromeritics, USA).

The dispersion of the ceramic pigment was tested using Na-Octanoate as an anionic surfactant and polyacrylic acid (Na-PAA, MW. $15000 \mathrm{~g} / \mathrm{mol}$ ) containing $\mathrm{Na}^{+}$salt as an anionic polymer electrolyte. Each of the dispersants was added at a concentration in a range of 0 to $2.0 \mathrm{mg} / \mathrm{m}^{2}$ with reference to the surface area of the ceramic pigment; the resulting solution was stirred for $2 \mathrm{~h}$ to reach chemical equilibrium. Then, the $\mathrm{CoAl}_{2} \mathrm{O}_{4}$ pigment was added to the resulting solution. The ceramic ink containing the ceramic pigment at $35 \mathrm{wt} \%$ was milled for $72 \mathrm{~h}$ using a zirconia medium. The dispersant showing the best pigment dispersion as well as its mixing ratio was determined by analyzing the flowability and the sedimentation behavior.

In addition, the applicability to the inkjet printing process 
was analyzed by varying the surface tension and the viscosity of the prepared ceramic ink by respectively using a polysiloxane-based additive (BYK-28, BYK) and poly ethylene oxide (PEO, MW. 100,000, 200.000, 400,000 g/mol). The viscosity of the ceramic ink was measured using a rotational rheometer (HAAKE MARS III, Thermo Fisher Scientific Inc.); the surface tension was measured using a surface tension analyzer (DST-60, Surface Electro Optics Co.). A drop watcher (STI Co.) was also used to analyze the drop formation behavior in inkjet printing depending on the properties of the ceramic ink.

\section{Results and Discussion}

Figure 1 shows the crystal structure and the microstructure of the $\mathrm{CoAl}_{2} \mathrm{O}_{4}$ ceramic pigment. The XRD result shown in Fig. 1(a) indicates that the crystal structure of the ceramic pigment included a spinel face corresponding to $\mathrm{CoAl}_{2} \mathrm{O}_{4}$, represented by the main peak, and the corundum face corresponding to $\mathrm{Al}_{2} \mathrm{O}_{3}$. The result of the pigment particle size and microstructure analysis, shown in the FE-SEM image in Fig. 1(b), indicates that the size of the primary particles of the pigment were about 200 to $300 \mathrm{~nm}$. Almost no coagulation was found between the pigment particles, and most of the particles were angular. The density and the specific surface area of the $\mathrm{CoAl}_{2} \mathrm{O}_{4}$ pigment were $4.44 \mathrm{~g} / \mathrm{cm}^{3}$ and $5.55 \mathrm{~m}^{2} / \mathrm{g}$, respectively, as shown in Table 1.

To secure the dispersion of the ceramic pigment, Na-Octanoate, an anionic surfactant, and Na-PAA, a polymer electrolyte dispersant, were respectively added to inks containing the $\mathrm{CoAl}_{2} \mathrm{O}_{4}$ ceramic pigment at $35 \mathrm{wt} \%$; the viscosity of the ink depending on the shear rate was measured, with results shown in Fig. 2. Regardless of the type and the mixing ratio of the dispersants, non-Newtonian behavior was found in all the samples with shear-thinning. As the content of the added dispersant increased, the viscosity of the ceramic ink slightly decreased.

When Na-Octanoate was used as a dispersant, the viscosity of the ceramic ink did not significantly decrease as the content of the added dispersant increased, which may be
Table 1. Properties of $\mathrm{CoAl}_{2} \mathrm{O}_{4}$ Pigment

\begin{tabular}{ccc}
\hline Diameter $(\mathrm{nm})$ & Density $\left(\mathrm{g} / \mathrm{cm}^{3}\right)$ & $\begin{array}{c}\text { BET surface area } \\
\left(\mathrm{m}^{2} / \mathrm{g}\right)\end{array}$ \\
\hline $200-300$ & 4.443 & 5.548 \\
\hline
\end{tabular}
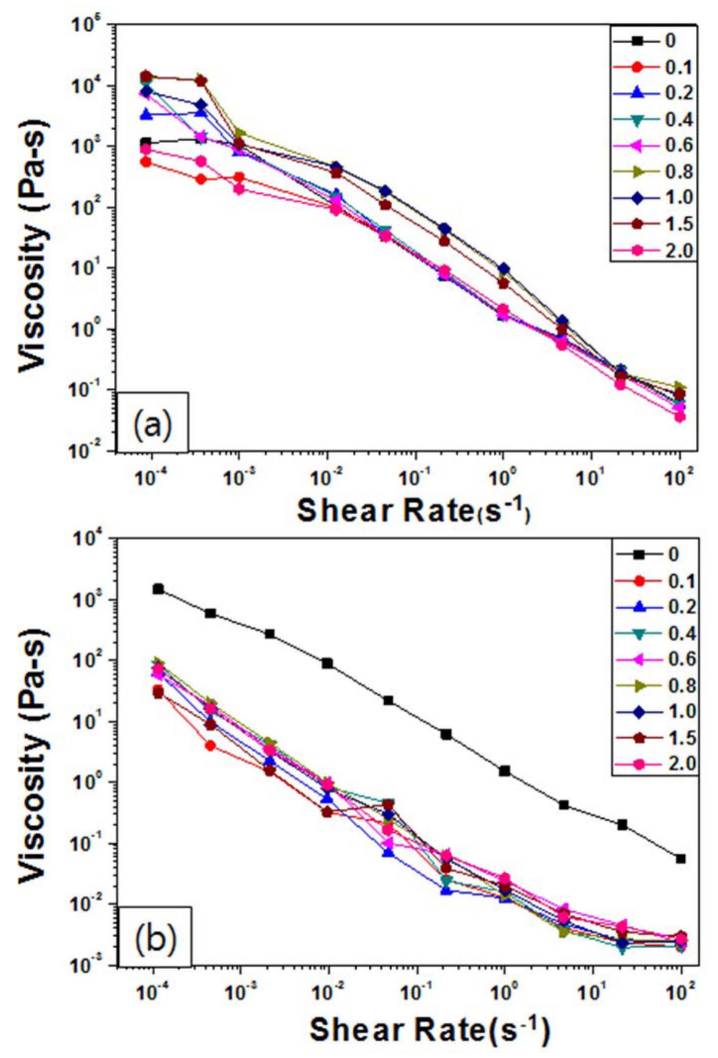

Fig. 2. Rheological behavior of $\mathrm{CoAl}_{2} \mathrm{O}_{4}$ suspensions with addition of (a) Na-Octanoate and (b) Na-PAA.

because the anionic surfactant was not well adsorbed on the pigment particle surface or even because the adsorbed surfactant failed to show sufficient repulsive force between the pigment particles, having little effect on the dispersion. On the contrary, when Na-PAA was used as a dispersant, the



Fig. 1. (a) X-ray diffraction pattern and (b) SEM image of $\mathrm{CoAl}_{2} \mathrm{O}_{4}$ pigment. 


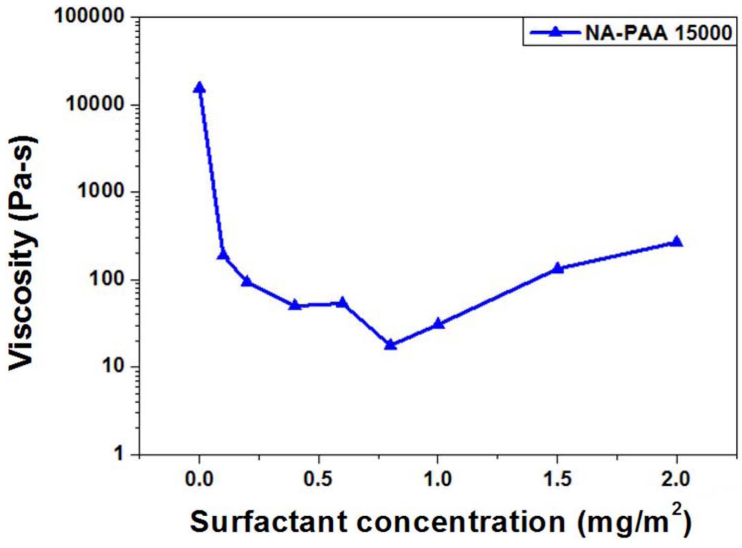

Fig. 3. Apparent viscosity of $\mathrm{CoAl}_{2} \mathrm{O}_{4}$ suspensions with dispersant concentration (numbers in the textbox indicate the molecular weights of polyelectrolytes used in this study).

viscosity significantly changed. Therefore, Na-PAA was selected as a more effective dispersant for the $\mathrm{CoAl}_{2} \mathrm{O}_{4}$ pigment. As the addition of the dispersant increased, the viscosity of the ceramic ink decreased, which indicates that
Na-PAA was effectively adsorbed to the $\mathrm{CoAl}_{2} \mathrm{O}_{4}$ pigment surface and thus the dispersion was probably stabilized by the stabilization mechanisms of electrostatic stabilization and steric hindrance. ${ }^{11)}$ Based on the results shown in Fig. 2(b), the apparent viscosity of the ceramic ink was measured depending on the content of the Na-PAA dispersant, as shown in Fig. 3. The dispersion of the ceramic pigment may have been most effective at the Na-PAA concentration of $0.8 \mathrm{mg} / \mathrm{m}^{2}$, with the lowest viscosity. ${ }^{12)}$

Figures 4 and 5 show the sedimentation behavior of the ceramic inks prepared using Na-Octanoate, which is an anionic surfactant, and Na-PAA, which is a polymer electrolyte dispersant, respectively. To prepare ceramic inks, each of the dispersants was added at a concentration in a range of 0 to $2.0 \mathrm{mg} / \mathrm{m}^{2}$ with reference to the surface area of the ceramic pigment. To analyze the dispersion stability, the sedimentation behavior was observed by keeping the ceramic ink in a measuring cylinder for two months. The flocculation of the ceramic pigment particles over time was investigated by measuring the volume of the bottom layer (sediment), middle layer (clarified), and top layer (float). Fig. 4 shows the sedimentation behavior of the ceramic ink
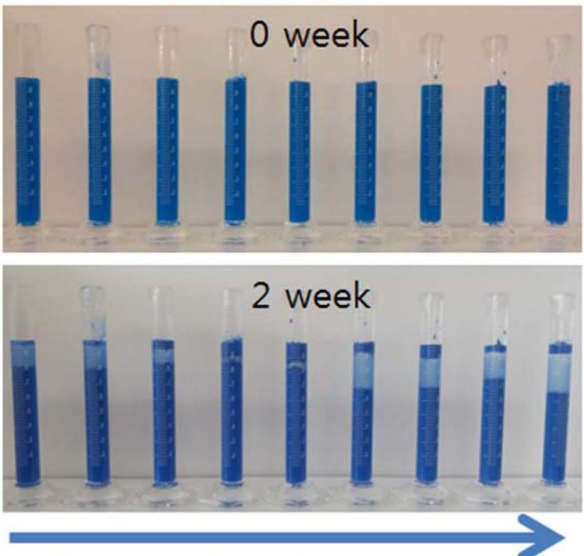

Surfactant load from 0 to $2.0 \mathrm{mg} / \mathrm{m}^{2}$
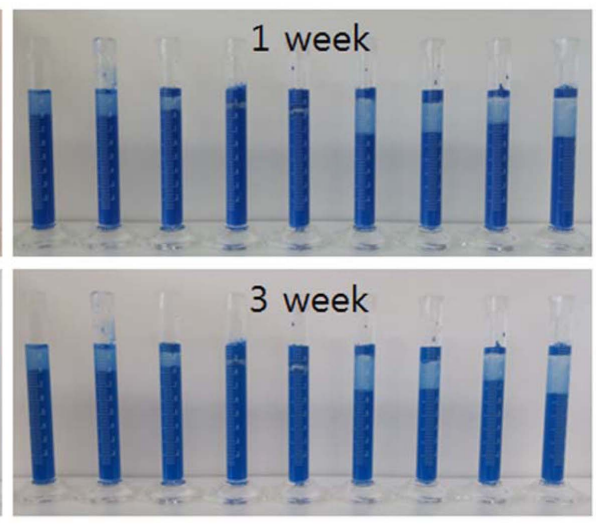

Fig. 4. Sedimentation behavior of $\mathrm{CoAl}_{2} \mathrm{O}_{4}$ suspensions dispersed with Na-Octanoate.


Surfactant load from 0 to $2.0 \mathrm{mg} / \mathrm{m}^{2}$
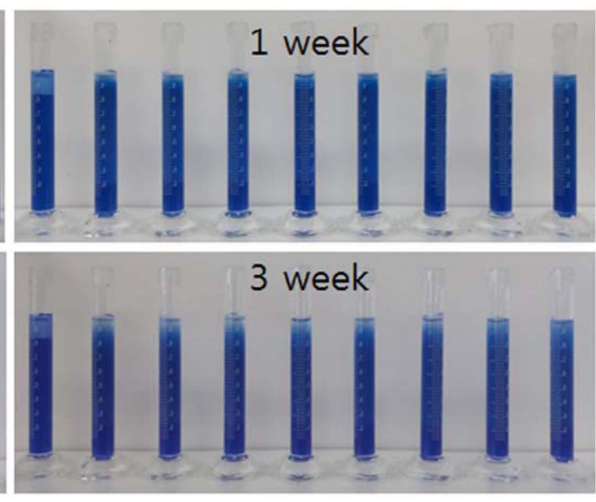

Fig. 5. Sedimentation behavior of $\mathrm{CoAl}_{2} \mathrm{O}_{4}$ suspensions dispersed with Na-PAA. 


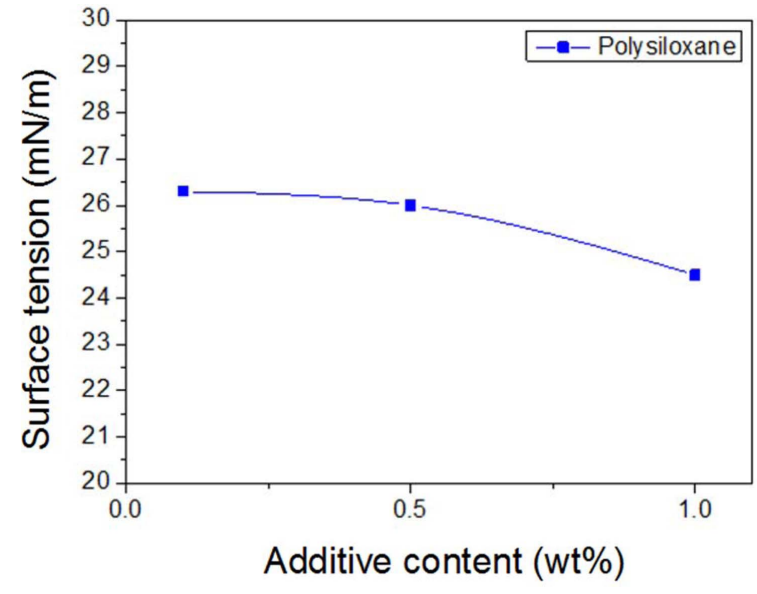

Fig. 6. Influence of additive concentration on surface tension of $\mathrm{CoAl}_{2} \mathrm{O}_{4}$ suspensions.

prepared using Na-Octanoate, which is an anionic surfactant; it can be seen that the top layer was clarified from one day after the preparation. As time passed, the layers separated further. Such stratification may have been caused by the flocculation of the $\mathrm{CoAl}_{2} \mathrm{O}_{4}$ particles, which have high surface energy in the ceramic ink, over time. Fig. 5 shows the sedimentation behavior of the ceramic ink prepared using Na-PAA, which is an anionic polymer electrolyte. The sedimentation test showed that the sedimentation of the ceramic ink containing the anionic polymer electrolyte occurred slowly over three weeks. This shows that the repulsive force between the particles caused by the polymer adsorbed to the particles and the anions surrounding the polymer may have prevented flocculation and sedimentation of the particles.

According to the optimal dispersion conditions mentioned above, the ceramic pigment was dispersed by adding NaPAA at the concentration of $0.8 \mathrm{mg} / \mathrm{m}^{2}$; a polysiloxane-based additive to control the surface tension of the ceramic ink was added at $0.1 \mathrm{wt} \%, 0.5 \mathrm{wt} \%$, and $1.0 \mathrm{wt} \%$ with reference to the pigment contained in the ink; then, the surface tension was measured, with results shown in Fig. 6. Control of the surface tension and the viscosity is a necessary factor for ceramic inks used for digital printing. In the preparation of ceramic inks, one of the important characteristics of a water-based system is that the high surface tension gives capillary force to the nozzle when jetting the ink. At the moment when a drop is separated from the nozzle, the surface area is instantaneously reduced to make the drop spherical, and the tail of the drop is quickly shortened. Therefore, in the smooth jetting of a water-based ceramic ink, it is critical to control the surface tension of the waterbased ceramic ink within the appropriate range of 20 to 45 $\mathrm{mN} / \mathrm{m}$, as required in inkjet printing. The surface tension of the ink was $64 \mathrm{mN} / \mathrm{m}$ before the addition of polysiloxane, which is a surface tension modifier, and 26.3, 26.0, and 24.5 $\mathrm{mN} / \mathrm{m}$ when the polysiloxane content was increased to 0.1 wt $\%, 0.5 \mathrm{wt} \%$, and $1.0 \mathrm{wt} \%$, respectively. As the mixing ratio of polysiloxane increased, the surface tension decreased. The surface tension appropriate for an inkjet printing process was achieved by adding just $0.1 \mathrm{wt} \%$ polysiloxane.

Figures 7 and 8 show the drop formation behavior of the ceramic ink as it depends on the surface tension and viscosity. All the ceramic inks were prepared by adding as a dispersant Na-PAA and polyethylene oxide (PEO, Mw. 100,000, 200,000 , and $400,000 \mathrm{~g} / \mathrm{mol}$ ) of different molecular weights at $2 \%$; then, the jetting characteristics were analyzed.

Figure 7 shows the drop formation of the ceramic ink with unadjusted surface tension. A convex meniscus was formed at 0 to $20 \mu \mathrm{s}$; the drop was elongated by the transferred

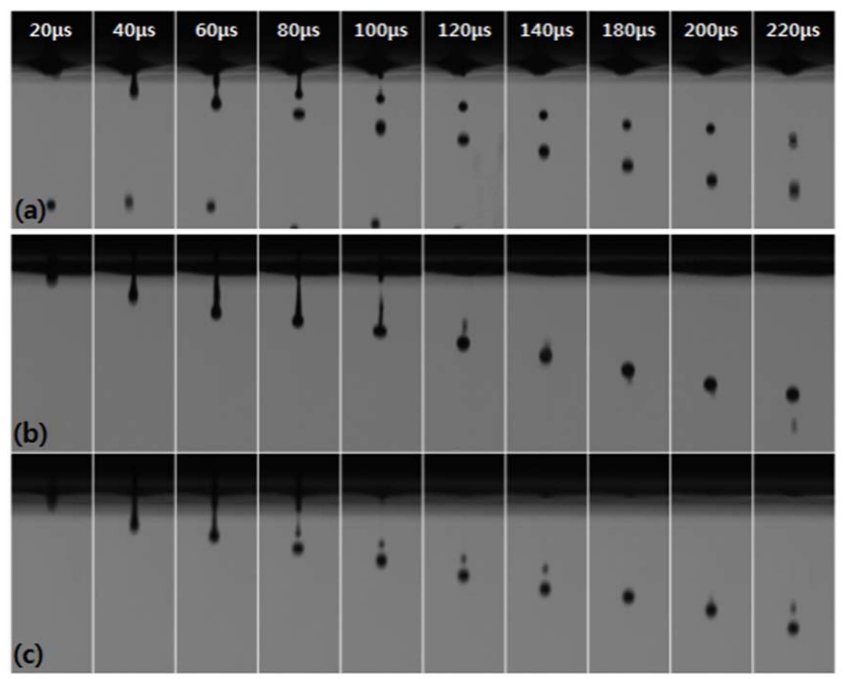

Fig. 7. Drop formation behavior of ceramic inks with addition of viscosity modifier: (a) PEO (Mw. 100,000), (b) PEO (Mw. 200,000), (c)PEO (Mw. 400,000).
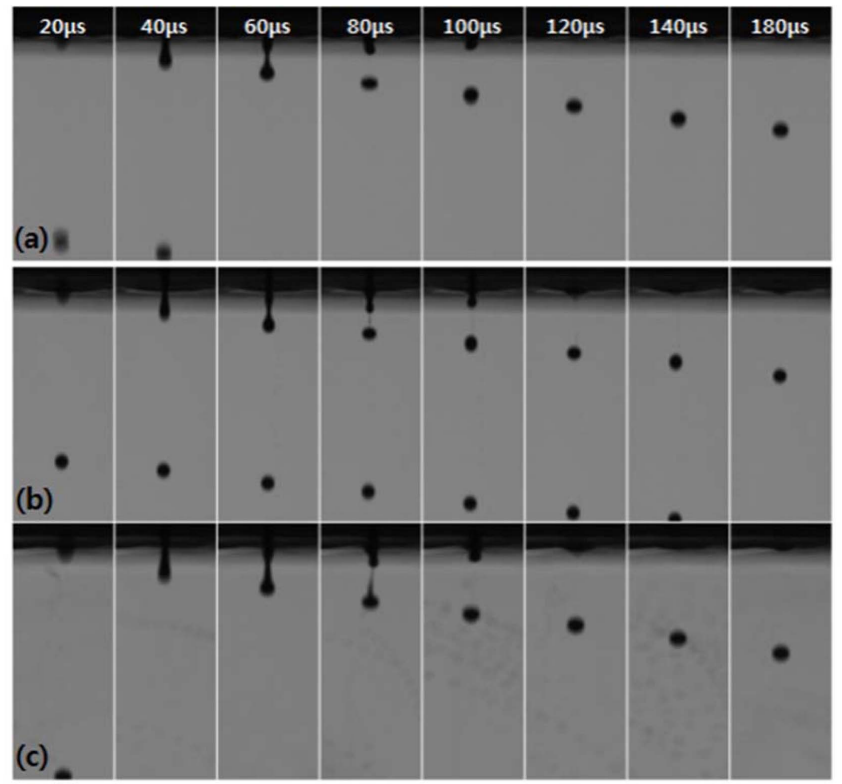

Fig. 8. Drop formation behavior of ceramic inks with addition of viscosity modifier: (a) PEO (Mw. 100,000), (b) PEO (Mw. 200,000), (c) PEO (Mw. 400,000), and surface tension modifier. 



Fig. 9. Pattern printing on ceramic tiles using ceramic inks formulated (a) without surface tension modifier and (b) with surface tension modifier.

pressure vibration at 40 to $60 \mu \mathrm{s}$; it then separated from the original drop at $80 \mu \mathrm{s}$. Due to the high surface tension, which tends to minimize the surface area of an elongated tail and drop, high tension acts inside the drop, separating individual drops and forming satellite drops. As the viscosity of the ink was increased by the addition of PEO to 4.35 , 5.43 , and $12.9 \mathrm{mPa} \cdot \mathrm{s}$, the distance between the original drop and the satellite drop was reduced.

Figure 8 shows the results obtained by lowering the surface tension of the water-based ceramic ink from $62 \mathrm{mN} / \mathrm{m}$ to $32.5 \mathrm{mN} / \mathrm{m}$ by adding polysiloxane $0.1 \%$. Analysis of the jetting behavior showed that a meniscus was formed at 0 $20 \mu \mathrm{s}$; the drop was elongated at $40-60 \mu \mathrm{s}$; then, the drop separated as a single independent drop at $80 \mu \mathrm{s}$. When the surface tension was controlled, stable jetting was found under all conditions.

To verify the applicability to inkjet printing, inkjet printing was performed on ceramic tiles using a ceramic ink with uncontrolled surface tension to form satellite drops in jetting (Fig. 7(c)); another ceramic ink with controlled surface tension showed stable jetting characteristics (Fig. 8(c)), as shown in Fig. 9. Fig. 9(a) shows that the ceramic ink having uncontrolled surface tension formed satellite drops, not a single drop, at the moment of ink jetting, resulting in printing at positions not in the desired pattern. This may cause low printing resolution. On the contrary, Fig. 9(b) shows that a single spherical drop formed when the viscosity and surface tension were controlled to be appropriate for the printing requirements. In addition, the results of printing with jetted ceramic ink showed that overlap between printed drops was minimized, and only the desired pattern was printed at the right position, as required by the user.

\section{Conclusions}

In the present study, a water-based ceramic ink was synthesized and applied to inkjet printing. The microstructure and the crystal structure of the selected ceramic pigment, $\mathrm{CoAl}_{2} \mathrm{O}_{4}$, were analyzed. For application to inkjet printing, the viscosity and the surface tension of the ceramic ink were properly controlled, and the applicability to inkjet printing and the drop formation behavior were analyzed. The $\mathrm{CoAl}_{2} \mathrm{O}_{4}$ pigment, having a particle size in a range of 200 to $300 \mathrm{~nm}$, was dispersed using Na-PAA (Mw. 15,000 g/mol), which is an anionic polymer electrolyte. The best dispersion was found at the dispersant content of $0.8 \mathrm{mg} / \mathrm{m}^{2}$. In addition, the jetting characteristics of the ink were analyzed by optimizing the viscosity and the surface tension. For the printing of a clearer pattern, formation of satellite drops in the drop formation process was minimized in the inkjet printing process on ceramic tiles. This showed that the water-based ceramic ink prepared in the present study may be applied to digital printing processes.

\section{Acknowledgements}

This study was supported by the Global Professional Technology Development Project (10059003) of the Ministry of Trade, Industry and Energy.

\section{REFERENCES}

1. M. T. Cavalcante, M. Dondi, G. Guarrini, M. Raomondo, and G. Baldi, "Colour Performance of Ceramic Nanopigments," Dyes Pigm., 80 [2] 266-68 (2006).

2. D. Gardini, F. Matteucci, M. Blosi, A. L. Costa, M. Dondi, C. Galassi, M. Raimondo, G. Baldi, and E. Cinotti, "ChemicoPhysical Properties of Nano-Sized Ceramic Inks for InkJet Printing," Qualicer 2006-IX Global Forum on Ceramic Tile., 397-410 (2006).

3. W. Eitel, Silicate Science; Vol. 1, pp. 149-55, Academic Press, New York, 1964.

4. A. L. Bowman and N. H. Krikorian, "Interstitial Phases," pp. 253-92 in Treatise on Solid State Chemistry, Vol. 3, Crystalline and Noncrystalline Solids. Ed. by N. B. Hannay, Academic Press, London, 1983.

5. M. Dondi, M. Blosi, D. Gardini, and C. Zanelli, "Ceramic Pigments for Digital Decoration inks: An Overview," Ceram. Forum. Inter., 89 [1] 1-11 (2012)

6. R. Morene, "The Role of Slip Additives in Tape-Casting Technology: Part I- Solvents and Dispersant," Am. Ceram. 
Soc. Bull., 71 [10] 1521-23 (1992)

7. P. Calvert, "Inkjet Printing for Materials and Devices," J. Am, Ceram. Soc., 13 [10] 3299-301 (2001)

8. H. R. Kang, "Water-based Ink-Jet Ink. I, Formulation," J. Imaging. Sci., 35 [3] 179-88 (1991).

9. Z. Q. Lin, J. Liang, P. J. Sun, F. Liu, Y. Y. Tay, M. D. Yi, K. Peng, X. H. Xia, and L. H. Zhou, "Spirocyclic Aromatic Hydrocarbon-based Organic Nanosheets for Eco-friendly Aqueous Processed Thin-Film Non-Volatile Memory Devices," Adv, Mater., 25 [27] 3663 (2013).
10. W. J. Lee, H. J. Hwang, K. S. Han, W. S. Cho, and J. H. Kim, "Characterization and Synthesis of Aqueous Pinkred Ceramic Ink for Digital Inkjet Printing," J. Cryst. Growth., 25 [1] 20-2 (2015)

11. M. J. Park, J. W. Ahn, and H. Kim, "Study on the Dispersion Stability of Precipitated Calcium Carbonate Suspensions," J. Korean Ceram, Soc., 38 [1] 343 (2001).

12. R. E. Mistler and E. R. Twiname, "Tape Casting : Theory and Practice," J. Am. Ceram. Soc., 64 [9] 7-62 (2000). 